\title{
Evaluation of collaborative TB/HIV activities in a general hospital in Addis Ababa, Ethiopia
}

Aragie Kassa ${ }^{*}$, Degu Jerene ${ }^{2}$, Yibeltal Assefa ${ }^{2}$, Azmera Teka ${ }^{3}$, Abraham Aseffa $^{4}$ and Amare Deribew ${ }^{5}$

\begin{abstract}
Background: Ethiopia has had mechanisms for TB/HIV collaborative activities since 2002. However, no published account has defined the role of these collaborative efforts in strengthening linkages between HIV and TB management units at the point-of-care level. Our objective was to assess the extent of linkages between the two programs at the patient management level at Zewditu Memorial Hospital in Addis Ababa, Ethiopia. Between January and December 2008, the registers of 241 TB patients were reviewed to determine the HIV testing rate, the treatment charts of 238 randomly selected patients were reviewed for providers' compliance with evaluation criteria, and exit interviews were conducted with 309 TB/HIV co-infected clients to validate providers' compliance.

Results: From register review, it was determined that the HIV testing acceptance rate was $95 \%$, and that $70 \%$ of patients received post-test counseling. A review of the patient chart revealed that of 51 patients with a complaint of cough, duration for cough was recorded in 35 (68.6\%) cases and cough > 2 weeks was recorded in 25 (49.0\%) cases. Seventy two percent (18 of 25) were linked for sputum microscopy. Linkage to cotrimoxazole prophylactic treatment was $81 \%$, but only $47 \%$ of eligible patients were linked to isoniazid preventive therapy (IPT). Correct diagnosis was accomplished at a rate of $100 \%$ for smear positive pulmonary TB, $23 \%$ for smear negative pulmonary TB and 88\% for extra pulmonary TB patients. Both chart review and exit interviews indicated that history of TB contact and cough $>2$ weeks predicted TB disease.
\end{abstract}

Conclusion: The rates of HIV testing and linkage to cotrimoxazole prophylactic therapy were high. Improvement is needed in the areas of recording patient information, screening HIV positives for TB, initiation of IPT, referral, linkages, and TB diagnostic capacity.

\section{Background}

Tuberculosis (TB) is the leading killer of human immunodeficiency virus (HIV) infected individuals, and the overlapping epidemics have had a devastating impact on TB and HIV morbidity, mortality and control worldwide [1]. These TB and HIV co-epidemics require urgent and effective attention and demand a collaborative effort between TB and HIV programs employing different but complementary strategies. Both programs should be able to identify and manage both diseases $[2,3]$.

However, the two programs are often separate at the level of patient care contributing to delayed diagnosis and linkage to care. TB and HIV programs must establish linkages to better utilize resources, avoid missed opportunities, and accelerate universal access to

\footnotetext{
* Correspondence: aragiek@gmail.com

${ }^{1}$ Ministry of Health, Addis Ababa, Ethiopia

Full list of author information is available at the end of the article
}

comprehensive TB and HIV prevention, treatment and care services [4].

In Ethiopia, 24\% HIV sero prevalence is reported in TB patients from health facilities implementing TB/HIV services [5]. In 2000, of 51 consecutive cultures of proven TB patients in Addis Ababa, $47 \%$ were HIV Positive [6]. Nonetheless, no research in Ethiopia defined the collaborative activities since it started implementation in 2002. The objective of this study was to describe the level of linkage between TB and HIV programs in patients attending care and treatment in Zewditu Memorial Hospital in Addis Ababa.

\section{Methods}

Study setting

Zewditu Memorial Hospital (ZMH) is a general hospital located in Addis Ababa, Ethiopia. It has been providing antiretroviral therapy since 2003. Addis Ababa has a
Ciomed Central

(C) 2012 Kassa et al; licensee BioMed Central Ltd. This is an Open Access article distributed under the terms of the Creative Commons Attribution License (http://creativecommons.org/licenses/by/2.0), which permits unrestricted use, distribution, and reproduction in any medium, provided the original work is properly cited. 
projected population of 3 million, and is divided in to 10 administrative sub cities and 99 localities [7,8].

As a standard of care, the Ethiopian national guidelines for TB/HIV collaboration advised offering HIV testing to those with cough $>2$ weeks, provision of IPT to HIV positives screened negative for TB, cotrimoxazole prophylactic therapy (CPT) to co- infected patients, and regular TB screening of clients in HIV care [5].

\section{Study design and data collection}

This cross-sectional study was conducted between January and December 2008. Three methods were used to evaluate the performance of the TB/HIV collaborative activities: review of registers of TB patients to determine the HIV testing rate, retrospective review of charts of patients coinfected with TB and HIV, and exit interviews. The registers of all the $241 \mathrm{~TB}$ patients who visited the clinic between January and December 2008 were reviewed, as well as the charts of 238 randomly selected TB/HIV coinfected patients aged 18 years and above and treated at the $\mathrm{ZMH}$ during the specified period. In addition, 309 randomly selected TB/HIV co-infected patients were interviewed during the data collection period.

Information was retrieved from $\mathrm{TB}$ and providerinitiated counseling and testing (PICT) registers and from HIV care/anti-retroviral treatment (ART) charts using a data abstraction checklist. Data from registers and charts included information on HIV testing and post test counseling of TB patients, referral to HIV care of HIV positive TB patients, screening of HIV positives for (cough, fever, and night sweating $>2$ weeks), weight lost $>3 \mathrm{~kg}$ in the past 4 months, and history of TB contact in the past 1 year, sputum microscopy for acid-fast bacilli (AFB), sputum culture, lymph node fine needle aspiration (FNA) test, linkage to IPT and CPT. Structured questionnaire containing standard TB screening criteria(if the client had cough, fever, and night sweats for $>2$ weeks, weight loss $>3 \mathrm{~kg}$ in the last 4 weeks, and $\mathrm{TB}$ contact in the past 1 year) were used for exit interview of clients how they screened for TB. The groups of patients included in the exit interview were those screened for TB in the past 1 year.

The data abstraction checklist and the exit interview questionnaire were adapted from the national TB/HIV guideline [5], and pretested before being applied for data collection. The questionnaire for exit interview was translated to Amharic (local language) and translated in English to maintain consistency. We used experienced and trained data collectors for all data collections activities.

\section{Evaluation criteria}

The following indicators adapted from World Health Organization (WHO) guidelines were used to evaluate the level of linkage implemented between TB and HIV services: TB patients who are tested for HIV, HIV positive clients given IPT, co infected patients put on CPT, proportion of patients with cough $>2$ weeks duration and sputum for AFB is ordered, proportions of TB suspect patients diagnosed correctly for TB $[5,9]$.

TB screening of HIV positives included asking questions based on a combination of symptoms of the five TB screening criteria indicated in the national protocol. The screening checklist included cough, fever, night sweats (> 2 weeks), weight loss $>3 \mathrm{~kg}$ in the last 4 weeks, and history of TB contact in the past 1 year. If the client said yes for cough $>2$ weeks or if no to cough $>2$ weeks but yes to two or more of the other questions the patient was further evaluated [5].

Diagnosis of TB in HIV positive patients was based on the national TB/HIV guidelines. Pulmonary TB (PTB) diagnosis was established if at least one sputum smearpositive for AFB was detected. The algorithm for smear negative $\mathrm{PTB}$ diagnosis required at least 2 slides negative for sputum AFB, no response to broad spectrum antibiotic for 10-14 days, and radiographic abnormalities consistent with active TB; or, at least 2 slides negative for sputum AFB plus sputum culture positive for M. tuberculosis. Extra pulmonary TB (EPTB) diagnosis was based on FNA suggestive of, or consistent with, active extra pulmonary $\mathrm{TB}$, one specimen from an extra pulmonary site culture positive for M.tuberculosis, or smear positive for AFB with the clinician's decision to treat the patient with a full course of anti-tuberculosis treatment.

\section{Statistical analysis}

Quantitative data was sorted, cleaned, and coded after double data entry using the EPI-Info version 3.3.2 data entry program. Analysis was conducted with SPSS 16.0 (SPSS inc. Chicago, 2007). Quantitative data was analyzed by calculating percentage of indicators using descriptive statistics. Percentages were used for comparison. We used both bivariate and multivariate analysis to estimate demographic characteristics and TB screening criteria for specific outcome variables in the program. We determined the level of significance at $P<0.05$.

\section{Ethical review}

The study was approved by the ethics committee of Addis Ababa Regional Health Bureau. The purpose of exit interview was described and participation was fully voluntary and all study subjects gave verbal consent. Interview was done privately and data captured from various sources were kept confidentially.

\section{Results}

HIV testing among TB patients

The mean age of registered TB patients was 30.2 years [range 18-80 years; standard deviation (SD) 12.9]. Of 
241 TB patients, 95\% were tested for HIV. About 86\% (68/79) of those tested positive received post-test counseling, $6 \%$ did not receive post-test counseling, and in about $8 \%$ the status of post-test counseling was missing (Table 1).

\section{Medical chart reviews for provider compliance}

Mean age of patients whose charts were reviewed was 34.6 years (range 18-60; SD 8.1). With respect to educational attainment, $42.8 \%$ had completed primary school and $57.1 \%$ had completed secondary and above. About $46.2 \%$ were unemployed.

From 106 patients on ART and 132 patients on preART category included in the study, 44(41.5\%) and 89 (67.4\%) of patients were diagnosed for any form of TB respectively. Two hundred and four (85.7\%) patients were asked for a history of cough. Fifty-one patients (25.0\%) had complaint of cough and duration of cough was recorded for $35(68.6 \%)$. Cough lasting $>2$ weeks was recorded for $25(49.0 \%)$ of patients and $18(72.0 \%)$

Table 1 Baseline characteristics of the study participants in Zewditu Memorial Hospital, Dec 2008

\begin{tabular}{|c|c|c|}
\hline Characteristics & & $\begin{array}{l}\text { Number } \\
(\%)\end{array}$ \\
\hline \multicolumn{3}{|l|}{ Gender } \\
\hline & Female & 125(51.9) \\
\hline & Male & $116(48.1)$ \\
\hline \multicolumn{3}{|l|}{ Age } \\
\hline & $18-30$ & 156(64.7) \\
\hline & $31-45$ & $59(24.5)$ \\
\hline & $46-80$ & $26(10.8)$ \\
\hline \multicolumn{3}{|c|}{ TB patients offered HIV testing } \\
\hline & Yes & 230(95.4) \\
\hline & No & $11(4.6)$ \\
\hline \multicolumn{3}{|l|}{ HIV test result } \\
\hline & Positive & 79(34.3) \\
\hline & Negative & 123(53.5) \\
\hline & $\begin{array}{l}\text { Not } \\
\text { indicated }\end{array}$ & $28(12.2)$ \\
\hline \multicolumn{3}{|c|}{$\begin{array}{l}\text { Post test counseling for those tested for } \\
\text { HIV }\end{array}$} \\
\hline & Yes & 162(70.4) \\
\hline & No & 23(10.0) \\
\hline & $\begin{array}{l}\text { Not } \\
\text { indicated }\end{array}$ & 45(19.6) \\
\hline \multicolumn{3}{|c|}{ Referred sero-positives for HIV care } \\
\hline & $\begin{array}{l}\text { Within } \\
\text { facility }\end{array}$ & 25(31.6) \\
\hline & Other facility & $30(38.0)$ \\
\hline & $\begin{array}{l}\text { Not } \\
\text { indicated }\end{array}$ & 24(30.4) \\
\hline
\end{tabular}

were linked for investigation of sputum for AFB. The rate of sputum AFB positive for cough lasting $>2$ weeks was $29.6 \%$ with at least one slide positive for AFB. Of all the charts with diagnosis of TB, 27(11.3\%) were diagnosed for PTB, 65 (27.3\%) smear negative PTB, and 41 (17.2\%) were diagnosed for ЕРTB (Figure 1).

By chart review, one hundred and sixty three patients were symptom screen positive for fever and history of $\mathrm{TB}$ contact during the past year. Among these, 4(2.5\%), 65 (39.9\%), and 94(53.3), were not linked for radiographic examination, sputum test for AFB, and physical examination for further evaluation, respectively. For 171 patients who said "yes" for history of night sweating and TB contact at the same time, 4(2.3\%), 70(40.9\%), and 97 $(56.7 \%)$ were not linked to radiographic examination, sputum test for AFB, and physical examination for additional evaluation of tuberculosis, respectively. About $14.3 \%$ of patients included in the chart review were not screened for history of TB contact in the past year.

Correct diagnosis was done at the rate of $100 \%$ for smear positive PTB, $23 \%$ for smear negative PTB and $87.8 \%$ for ЕРTB patients. In multivariate analysis history of TB contact in the past 1 year $(96.3 \%$ for smear positive PTB, $90.8 \%$ for smear negative PTB and $92.7 \%$ for EPTB) and cough $>2$ weeks were predictors of TB disease (Table 2).

\section{Findings from the exit interview}

A total of 309 respondents participated in the exit interview. The mean age of the participants was 34 years (range 18-55; SD = 6.4); $77.7 \%$ were aged < 39 years; and $57 \%$ were females. $63.8 \%$ of respondents were secondary and above level of education and $30.4 \%$ of them were unemployed. Over a half $(52.1 \%)$ of the respondents were co-infected with TB in the past 3 years after they knew their HIV sero status of which 103(64.0\%) were taking CPT. Out of 162 co-infected patients 99 (61\%) have a history of TB contact in the past 1 year. From the multivariate analysis, history of TB contact in the past year was a major predictor of TB disease (Table 3). About $46.6 \%$ of interview participants were not screened for history of TB contact in the past year.

\section{Discussion}

This study uncovered high rates of HIV testing and linkage to CPT among TB patients. This can likely be attributed to the scaled up of PICT implementation in the study setting and show commendable progress [10]. However, the low IPT uptake, incompleteness of TB and PICT registers, and inadequate TB diagnostic capacity need more attention.

Although cough and history of TB contact in the past year were found to be a major predictors for symptom screening of tuberculosis in chart review (Table 2) and 


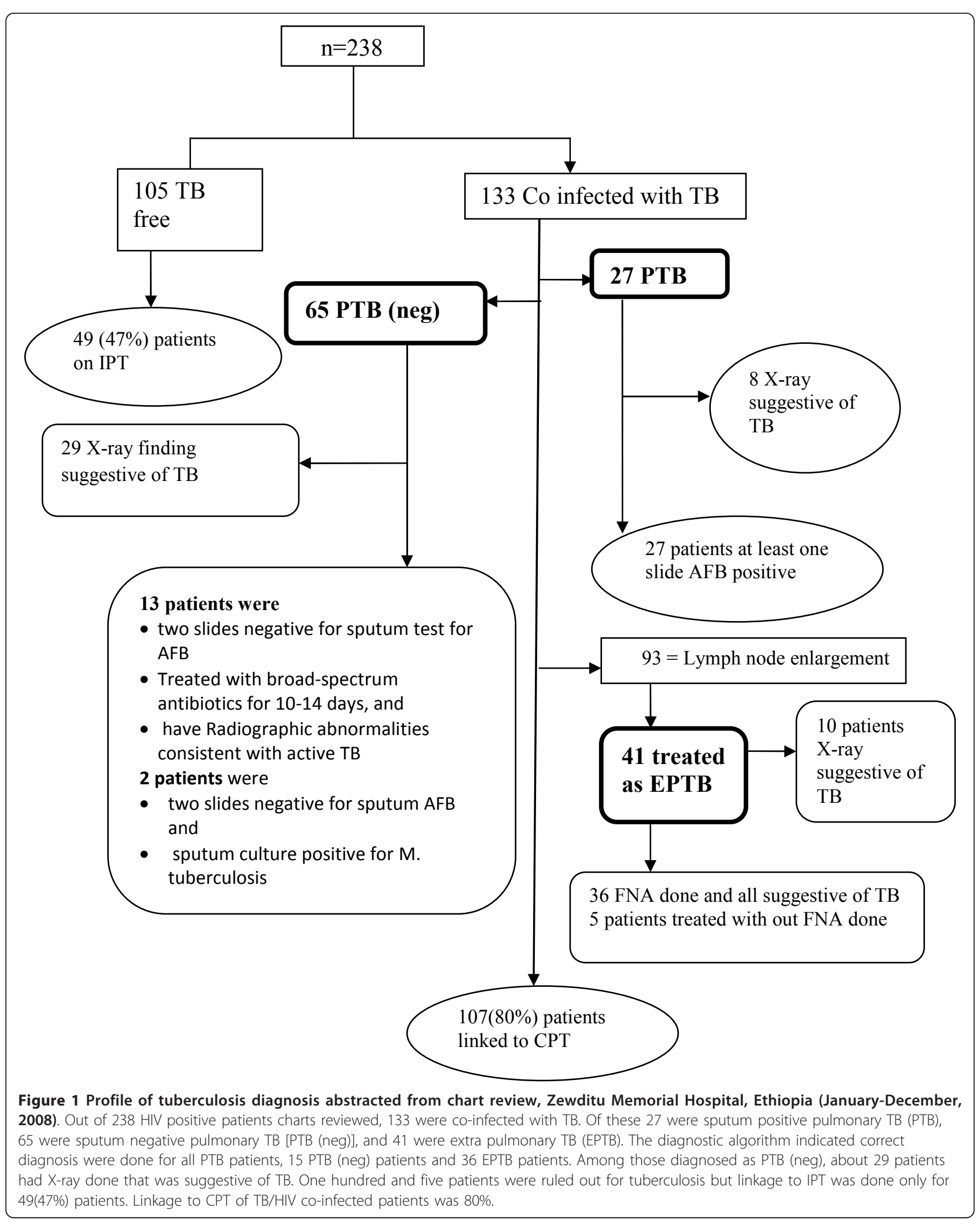


Table 2 Potential predictors of TB infection for chart review, Zewditu Memorial Hospital, Dec 2008.

\begin{tabular}{|c|c|c|c|c|c|}
\hline Predictor variable & Category & Crude OR & $P$-value* & Adjusted OR & $P$-value \\
\hline \multirow[t]{2}{*}{ Age } & $18-34$ & Referent & 0.31 & Referent & \\
\hline & $\geq 35$ & $\begin{array}{l}1.27 \\
(0.76-2.11)\end{array}$ & & $\begin{array}{l}1.22 \\
(0.71-2.07)\end{array}$ & 0.47 \\
\hline \multirow[t]{2}{*}{ Occupation } & Unemployed & Referent & & Referent & \\
\hline & Employed & $\begin{array}{l}0.96 \\
(0.74-1.26)\end{array}$ & 0.86 & $\begin{array}{l}0.82 \\
(0.48-1.42)\end{array}$ & 0.32 \\
\hline \multirow[t]{2}{*}{ Education } & No education & Referent & & Referent & \\
\hline & Primary \& above & $\begin{array}{l}0.77 \\
(0.48-1.31)\end{array}$ & 0.26 & $\begin{array}{l}1.62 \\
(0.63-4.17)\end{array}$ & 0.32 \\
\hline \multirow[t]{2}{*}{ History of TB contact } & No & Referent & & Referent & \\
\hline & Yes & $\begin{array}{l}3.06 \\
(1.36-6.89)\end{array}$ & 0.007 & $\begin{array}{l}2.81 \\
(1.22-6.45)\end{array}$ & 0.02 \\
\hline \multirow[t]{2}{*}{ Cough $>2$ weeks } & No & Referent & & Referent & \\
\hline & Yes & $\begin{array}{l}3.79 \\
(1.46-9.86)\end{array}$ & 0.006 & $\begin{array}{l}3.77 \\
(1.44-9.87)\end{array}$ & 0.01 \\
\hline
\end{tabular}

*Level of significance at $p<0.05$

in exit interviews (Table 3) respectively, both predictors were not consistently used as TB screening criteria. Poor adherence to the national guidelines in treating smear negative PTB was discovered, with less than one quarter of patients being diagnosed correctly. The same was true for EPTB patients.

The World Health Organization (WHO) recommends routine offering of HIV testing and CPT to all HIV patients co-infected with TB. CPT is recommended to all HIV positives co-infected with TB [3]. In a setting with significant challenges HIV testing and CPT rates of 95 and $81 \%$ respectively concurs with these global recommendations. Also, the HIV testing rate supersedes that reported by neighboring Kenya [11]. The CPT

Table 3 Analysis of TB contact as potential predictor to TB disease for exit interview of PLHIV, Zewditu Memorial Hospital, Dec 2008.

\begin{tabular}{|c|c|c|c|c|c|}
\hline $\begin{array}{l}\text { Predictor } \\
\text { variable }\end{array}$ & Category & $\begin{array}{l}\text { Crude } \\
\text { OR }\end{array}$ & $\begin{array}{l}P \text { - } \\
\text { value* }\end{array}$ & $\begin{array}{l}\text { Adjusted } \\
\text { OR }\end{array}$ & $\begin{array}{l}P \text { - } \\
\text { value }\end{array}$ \\
\hline \multirow[t]{2}{*}{ TB contact } & No & Referent & & Referent & \\
\hline & Yes & $\begin{array}{l}1.93 \\
(1.23-3.03)\end{array}$ & 0.004 & $\begin{array}{l}2.76 \\
(1.22-6.39)\end{array}$ & 0.006 \\
\hline \multirow[t]{2}{*}{ Age } & $18-34$ & Referent & & Referent & \\
\hline & $\geq 35$ & $\begin{array}{l}0.94 \\
(0.42-1.55)\end{array}$ & 0.81 & $\begin{array}{l}1.04 \\
(0.63-1.71)\end{array}$ & 0.87 \\
\hline \multirow[t]{2}{*}{ Occupation } & Unemployed & Referent & & Referent & \\
\hline & Employed & $\begin{array}{l}0.89 \\
(0.46-1.72)\end{array}$ & 0.61 & $\begin{array}{l}1.095 \\
(0.68-1.75)\end{array}$ & 0.71 \\
\hline \multirow[t]{2}{*}{ Education } & $\begin{array}{l}\text { No } \\
\text { education }\end{array}$ & Referent & & Referent & \\
\hline & $\begin{array}{l}\text { Primary \& } \\
\text { above }\end{array}$ & $\begin{array}{l}0.76 \\
(0.35-1.66)\end{array}$ & 0.492 & $\begin{array}{l}1.13 \\
(0.50-2.51)\end{array}$ & 0.77 \\
\hline
\end{tabular}

* Level of significance at $p<0.05$
Uptake in our study setting exceeds that documented in Uganda [12].

In concurrence with global reports (WHO 2010 report), the IPT uptake rate was low [13]. Although the guidelines recommend IPT as part of the standard care for HIV patients without active TB, low uptake continues to be challenge. This is not unique to Ethiopia. Fear of isoniazid (INH) resistance, failure to exclude active TB, poor adherence, shortage of supplies, and provider attitudes appear to impede its scaled up implementation [14]. This highlights the need to continue to advocate for improved delivery of IPT. More simplified guidelines are now available to guide its strengthened implementation [15]. Additional observed weaknesses of the program such as failure to utilize symptom screening, poor adherence to national guidelines with regard to treating smear negative PTB and EPTB can be addressed through continuous education and mentoring of health care providers.

Culture for Mycobacterium tuberculosis increases the likelihood of diagnosis [16]. Nonetheless, due to costs of sputum culture and FNA the proportion of patients benefited from both tests was very low. About $56.5 \%$ people living with HIV included in the study were unemployed and hence unable to make payments for the indicated laboratory investigations. A recent prospective study evaluating the prevalence of active TB in HIV infected patients with a CD4 cell count of $>200$ cells/ $\mu$ l reported that $29 \%$ of those with culture- confirmed pulmonary TB had normal radiography and clinical examination [17].

It appears that payments related with sputum culture and FNA impede compliance with the national protocol and the TB/HIV linkage activities in the study setting. Exempting patients from payments associated with these 
tests can create conditions conducive to resolving existing diagnostic problems. There is a need to strengthen laboratories with better TB diagnostic facilities, primarily for performing sputum culture and fluorescence microscopy. This could be done by assisting the existing laboratories in increasing the volume of testing and also providing financial support for them to conduct targeted tests such as sputum culture and FNA free of charge. Adding sputum culture to the package of care for people living with HIV (PLHIV) will substantially increase case detection, which is part of Ethiopia's commitment to improve TB and HIV care.

\section{Conclusion}

The high rates of HIV testing and linkages to CPT are encouraging indicators of progress in the implementation of TB/HIV collaborative activities in the study setting. However, low rates of IPT, poor diagnostic capacity, and inadequate documentation at TB clinics need to be addressed. Structural barriers to TB diagnostic facilities such as fees for TB culture need to be solved, and simpler diagnostic techniques should be made available in such settings. Periodic evaluation of the progress using larger sample sizes among nationally representative patient populations would contribute to improved quality of care to TB/HIV co-infected patients in Ethiopia.

\section{Acknowledgements}

The study was funded by Jimma University, school of Monitoring and Evaluation, in Ethiopia. We appreciate the city of Addis Ababa Health bureau administration for permitting us to conduct the study. We appreciate the participants for their good will to give the necessary information.

\section{Author details}

${ }^{1}$ Ministry of Health, Addis Ababa, Ethiopia. ${ }^{2}$ HIV/AIDS prevention and control office, Addis Ababa, Ethiopia. ${ }^{3}$ Private consultancy and research firm, Addis Ababa, Ethiopia. ${ }^{4}$ Armauer Hansen Research Institute, Addis Ababa, Ethiopia. ${ }^{5}$ Department of Epidemiology, Jimma University, Jimma, Ethiopia.

\section{Authors' contributions \\ AK was involved in the conception, design of the study, coordination of data collection, analysis, and drafting of the manuscript. AT was involved in design, field work, and review of the article. DJ, AD and YA participated in the design and review of the article. AA participated in the review of the article. All authors read and approved the final manuscript.}

\section{Competing interests}

The authors declare that they have no competing interests.

Received: 29 December 2010 Accepted: 26 January 2012

Published: 26 January 2012

\section{References}

1. WHO: Surveillance, planning, and financing. Global tuberculosis control. WHO/HTM/TB/2006.362 WHO; 2006.

2. Maher D, Floyd K, Raviglione M: A Strategic Framework to Decrease the Burden of TB/HIV Geneva: World Health Organization; 2002.

3. WHO: Stop TB Department and Department of HIV/AIDS. Interim policy on collaborative TB-HIV activities. Geneva, Switzerland: WHO; 2004.
4. Mukherjee JS: TB and HIV co-infection: initiating a comprehensive HIV prevention and treatment Program. The PIH guide to the communitybased treatment of HIV in resource-poor settings. U.S.A: Partners in Health; 2 2006, 18-26.

5. Federal Ministry of Health: Implementation Guidelines for TB/HIV collaborative Activities. Addis Ababa, Ethiopia. Ministry of Health; 2008.

6. Van den Hombergh J, Atnafu A, van Deutekom H: Chest X-ray and the diagnosis of PTB in an urban area with a high prevalence of HIV/TB Coinfection. Annual Medical Association Conference Addis Ababa, Ethiopia; 2001, 22-24, Abstract.

7. Addis Ababa Regional Health Bureau: Accelerated access to HIV/AIDS prevention, care and treatment in Addis Ababa Road Map. Addis Ababa, Ethiopia. Addis Ababa Health Bureau; 2006.

8. Ethiopian AIDS resource center:[http://www.etharc.org/resources/download/ view.download/42/225], Accessed 06, March 2008.

9. World Health Organization (2009): A guide to monitoring and evaluation for collaborative TB/HIV activities. Geneva, Switzerland: WHO; 2004.

10. United Nations General Assembly: Towards universal access: assessment by the Joint United Nations Programme on HIV/AIDS on scaling up HIV prevention, treatment, care and support. New York, NY, USA: UN General Assembly; 2006, A/60/737.

11. Odhiambo J, Kizito W, Njoroge A, Wambua N, Nganga L, Mburu M, Mansoer J, Marum L, Phillips E, Chakaya J, De Cock KM: Provider-initiated testing and counseling for TB patients and suspects in Nairobi, Kenya. Int J Tuberc Lung Dis 2008, 12(suppl3):63-68.

12. Okot-Chono R, Mugisha F, Adatu F, Madaraa E, Dlodlo R, Fujiwara P: Health system barriers affecting the implementation of collaborative TB-HIV services in Uganda. Int J Tuberc Lung Dis 2009, 13(8):955-961.

13. WHO: WHO report 2010. Global Tuberculosis Control Geneva, Switzerland: WHO; 2010.

14. Getahun H, Granich R, Sculier D, Gunneberg C, Blanc L, Nunn P, Raviglione M: Implementation of isoniazid preventive therapy for people living with HIV worldwide: barriers and solutions. AIDS 2010, 24(suppl 5): S57-65.

15. WHO: Department of HIV/AIDS and Stop TB department. Guidelines for intensified tuberculosis case-finding and isoniazid preventive therapy for people living with HIV in resource constrained settings Geneva, Switzerland: WHO; 2011.

16. Getahun $H$, Mark H, O'Brien R, Nunn P: Diagnosis of smear-negative pulmonary tuberculosis in people with HIV infection or AIDS in resource-constrained settings: informing urgent policy changes. Lancet 2007, 369:2042-2049.

17. Mtei L, Matee M, Herfort O, Bakari M, Horsburgh CR, Waddell R, Cole BF, Vuola JM, Tvaroha S, Kreiswirth B, Pallangyo K, Reyn CF: High rates of clinical and sub-clinical tuberculosis HIV-infected patients ambulatory subjects in Tanzania. Clin Infect Dis 2005, 40:1500-1507.

doi:10.1186/1756-0500-5-67

Cite this article as: Kassa et al:: Evaluation of collaborative TB/HIV activities in a general hospital in Addis Ababa, Ethiopia. BMC Research Notes 2012 5:67.

\section{Submit your next manuscript to BioMed Central and take full advantage of:}

- Convenient online submission

- Thorough peer review

- No space constraints or color figure charges

- Immediate publication on acceptance

- Inclusion in PubMed, CAS, Scopus and Google Scholar

- Research which is freely available for redistribution 